Polymer Journal, Vol. 6, No. 3, pp 242-247 (1974)

\title{
The Effect of Intramolecular Energy on the Conformation of Polymers by the Monte Carlo Methods
}

\author{
Yoshiro NAKATA* and Keizo SuZuKI \\ Department of Chemistry, Tokyo Kyoiku University, \\ Otsuka, Tokyo, Japan.
}

(Received November 14, 1973)

\begin{abstract}
The conformation of chain molecules is investigated by the "successive dimerization" technique. The three different models, the diamond lattice model, the GT-model of polyethylene and the modified GT-model of polyethylene, are used as the models for the polymer. The effect of intramolecular conformational energy is considered for the latter two models. A relation $\left\langle S^{2}\right\rangle=a N^{1.20}$ is obtained in all the models.
\end{abstract}

KEY WORDS Monte Carlo / Excluded Volume / Polyethylene / Random Walk / Conformation / Diamond Lattice /

The problem of the excluded volume effect in polymers has been treated by numerical methods using digital computers. The Monte Carlo calculation using a computer produces two kinds of problem.

(1) That of deciding which kind of model should be used to simulate the chain molecules. Most of the studies up to now have been performed by the restricted walks confined to a certain multidimensional lattice, but the relative stabilities of the real molecules due to intramolecular energy are not considered. Using a more realistic model, we estimate the intramolcular conformational energy for each conformation. The average dimensions are calculated by using the energy.

(2) That of discovering how the generation of a large number of nonintersecting random walks are accomplished. On the diamoned lattice, the number of steps in a walk $N$, which is attained in a reasonable number of samples, is a few hundreds by the direct Monte Carlo Methods. Wall and Erpenbeck ${ }^{1}$ therefore devised the "sample enrichment" technique. With the help of this method they constructed walks up to $N=600$ on the same lattice. Then, to obtain much longer walks, one of the present authors ${ }^{2}$

* Present address: Department of Chemistry; Faculty of General Study, Gunma University, Maebashi, Japan. devised the "successive dimerization" technique. The short self-avoiding walks are generated by the direct Monte Carlo Method, and are connected to each other as dimers. Two dimers are connected to give a tetramer, and two tetramers yields an octamer, and so on. By this method, 8192-step walks were obtained on a diamond lattice. However, for the more realistic model that takes the intramolecular energy into acount, a new extreme attrition has appeared according to the unbalanced weight by the Boltmann factor. For this reason, we could only construct a polyethylene chain with bond numbers up to 384 on the GT-model.

The Monte Carlo data could be well fitted by a relation of the form $\left\langle S_{N}{ }^{2}\right\rangle=a N^{b}$, where $\left\langle S_{N}{ }^{2}\right\rangle$ is the mean-square radius of gyration, and $a$ and $b$ are constants. The typical values of $b$ being 1.50 for a two-dimensional lattice model, and 1.20 for a three-dimensional lattice model. ${ }^{3}$ For the off-lattice model, at the present time it is reported only by E. Loftus and P. J. Gans ${ }^{4}$ that the value of $b$ is greater than the accepted values 1.20 for the lattice models. In the case of a very long chain, it is theoretically expected that the values of $b$ must be 1.20 for any threedimensional model.

In this paper the conformations of the model chain are studied by the successive dimerization technique of the Monte Carlo method with the intramolecular energy taken into account. The 
obtained values are discussed by comparing them with those of the diamond lattice model.

\section{MODEL}

The Monte Carlo calculation of nonintersecting random walks has been accomplished by specific lattice, nonlattice systems, etc. We then compare the three models, the diamond lattice model, the GT-model of polyethylene* and the modified GT-model of polyethylene. In the latter two models, the bond lengths and bond angles are fixed as shown in Table I. Three rotational isomeric states are chosen in the GT-model, i.e., trans $\left(180^{\circ}\right)$, gauche $\left(60^{\circ}\right)$, gauche $\left(300^{\circ}\right)$, and seven states are chosen in the modified GTmodel, i.e., trans $\left(175,180,185^{\circ}\right)$, gauche $(55$, $\left.65^{\circ}\right)$, gauche $\left(295,305^{\circ}\right)$; the angle for the cis form is taken to be $0^{\circ}$. The difference between the diamond lattice model and the GT-model lies in the existence of the hydrogen atoms,

Table I. Bondlengths and Bondangles

\begin{tabular}{cc}
\hline & Values \\
\hline $\mathrm{C}-\mathrm{C}$ & $1.54 \AA$ \\
$\mathrm{C}-\mathrm{H}$ & $1.08 \AA$ \\
$\angle \mathrm{CCC}$ & $109^{\circ} 28^{\prime}$ \\
$\angle \mathrm{CCH}$ & $109^{\circ} 28^{\prime}$ \\
$\angle \mathrm{HCH}$ & $109^{\circ} 28^{\prime}$ \\
\hline
\end{tabular}

although both chains have the same skeletal chains. A form of the GT-model of polyethylene is shown in Figure 1. It is drawn in perspective by the plotter system at the Computer Center of the University of Tokyo.

The well-known three-minima cosinusoidal rotational potential is replaced by a three minima square-well potential having energy zero in the trans states and $0.8 \mathrm{kcal} / \mathrm{mol}$ in the gauche states. It expresses the torsional energy. The nonbonded interactions are expressed by a Lennard-Jones " $6-12$ " potential

$$
U_{i j}=\frac{d_{i j}}{r_{i j}^{12}}-\frac{e_{i j}}{r_{i j}^{6}}
$$

The sets of constants ${ }^{6}$ in eq 1 are summarized in Table II.

Table II. The sets of potential function constants

\begin{tabular}{cccc}
\hline Pair & $d / \mathrm{kcal}^{12} \mathrm{~mol}^{-1}$ & $e / \mathrm{kcal}^{6} \mathrm{~mol}^{-1}$ & $r_{\text {min }}, \AA$ \\
\hline $\mathrm{H} \cdots \mathrm{H}$ & $4.5 \times 10^{3}$ & 47 & 2.4 \\
$\mathrm{C} \cdots \mathrm{C}$ & $286 \times 10^{3}$ & 370 & 3.4 \\
$\mathrm{C} \cdots \mathrm{H}$ & $38 \times 10^{3}$ & 128 & 2.9 \\
\hline
\end{tabular}

\section{THE METHOD OF CALCULATION}

A computer program embodying the model is written chiefly in FORTRAN IV and runs on

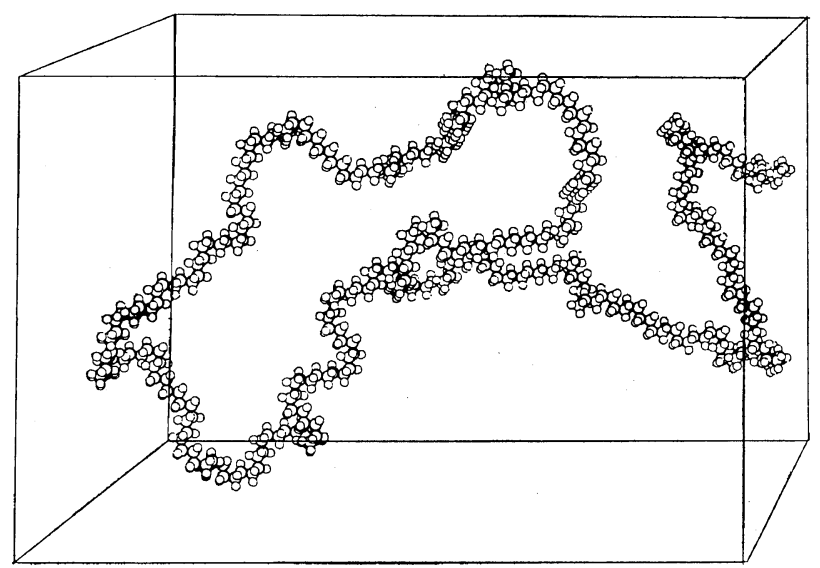

Figure 1. Schematic representation of a GT-model of Polyethylene: $N, 384 ; S^{2}, 1086 \AA^{2} ; R^{2}, 2664 \AA^{2}$.

\footnotetext{
* This model is explained fully in the previous paper. ${ }^{5}$
} 
the computer HITAC 5020E at Computer Center of the University of Tokyo.

The scheme of the program* to make chains without overlaps is as follows:

1 MAKE AN $M$-BOND CHAIN AT RANDOM.

2 IF THERE IS AN OVERLAP, GO TO 1.

$3 N=M$.

4 IF ANOTHER $N$-BOND CHAIN IS NOT STORED, STORE THE NEW CHAIN AND GO TO 1.

5 CONNECT THE STORED CHAIN TO THE NEW CHAIN AND MAKE A $2 N$ BOND CHAIN.

6 IF THERE IS AN OVERLAP, OUTPUT AND CLEAR BOTH THE $N$-BOND CHAINS, AND GO TO 1.

$7 N=2 N$, AND GO TO 4.

About the GT-model of polyethylene, the sterically allowed conformations are examined on the basis of the van der Waals radii.

\section{RESULTS AND DISCUSSION}

Random walks have been generated in the

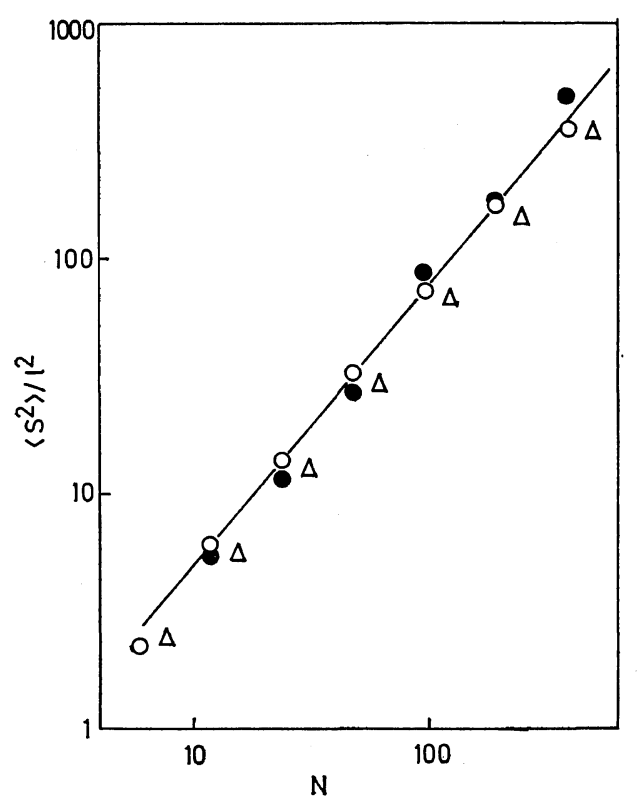

Figure 2. Values of $\log \left(S^{2} / l^{2}\right)$ vs. $\log N$ : ( $\left.\triangle\right)$, diamond lattice model; (O), GT-model; (O), mGTmodel.

Table III. Values of $S^{2} / l^{2 a}$

\begin{tabular}{|c|c|c|c|c|c|c|c|c|c|c|c|}
\hline \multicolumn{12}{|c|}{ GT-Model of polyethylene } \\
\hline$N$ & 6 & 12 & 24 & 48 & 96 & 192 & 384 & & & & \\
\hline$S^{2} / l^{2}$ & 2.277 & 6.114 & 13.79 & 32.51 & 73.03 & 170.5 & 351.5 & & & & \\
\hline$N_{\mathrm{s}}$ & & & & 2449 & 699 & 182 & 76 & & & & \\
\hline$\sigma$ & & & & 12.23 & 23.46 & 75.5 & 207.5 & & & & \\
\hline \multicolumn{12}{|c|}{ Modified GT-model of polyethylene } \\
\hline$N$ & 6 & 12 & 24 & 48 & 96 & 192 & 384 & & & & \\
\hline$S^{2} / l^{2}$ & 2.277 & 5.482 & 11.60 & 27.24 & 88.08 & 177.2 & 498.4 & & & & \\
\hline$N_{\mathrm{s}}$ & & & & 2462 & 702 & 150 & 70 & & & & \\
\hline$\sigma$ & & & & 7.84 & 21.12 & 24.6 & 184.6 & & & & \\
\hline \multicolumn{12}{|c|}{ Diamond lattice model } \\
\hline$N$ & 8 & 16 & 32 & 64 & 128 & 256 & 512 & 1024 & 2048 & 4096 & 8192 \\
\hline$S^{2} / l^{2}$ & 2.444 & 5.560 & 12.78 & 29.31 & 67.37 & 153.4 & 350.3 & 787.0 & 1740. & 3747. & 7943. \\
\hline$N_{\mathrm{s}}$ & & & & & & 4235 & 1072 & 256 & 307 & 102 & 38 \\
\hline$\sigma$ & & & & & & 65.0 & 145.9 & 325.0 & 797. & 1509. & 2666. \\
\hline
\end{tabular}

${ }^{a} l$, bond length; $T, 300^{\circ} \mathrm{K} ; N_{\mathrm{s}}$, number of samples; $\sigma$, dispersion.

diamond lattice model, the GT-model of poly- Mean Dimension ethylene and the modified GT-model of polyethylene by the successive dimerization technique.

\footnotetext{
* This scheme may "overflow" the capacity of the computer, and there should be an additional scheme, which treats the overflow.
}

Monte Carlo estimations of the mean-square radius of gyration $\left\langle S_{N}{ }^{2}\right\rangle$ are more precise than the mean-square end-to-end distance $\left\langle R_{N}{ }^{2}\right\rangle$. The values obtained to $\left\langle S_{N}{ }^{2}\right\rangle$ are given in Table III and the relation between $\left\langle S_{N}{ }^{2}\right\rangle$ and $N$ is shown 
in Figure 2. The results obtained for $\left\langle S_{N}{ }^{2}\right\rangle$ may be expressed by an equation of the form

$$
\left\langle S_{N}{ }^{2}\right\rangle=a N^{b}
$$

where $a$ and $b$ are constants.

On the diamond lattice model, where 8192 steps were attained, the exponent $b$ was quite close to 1.20 over the range $N>8$. The model in the range satisfies the postulate in the theories, which conclude that $b=1.20$ for all the threedimensional models. ${ }^{7}$

The range tends to recede towards larger $N$ in the bulky or stiff chains. However, the result on the GT-model shows the recession is small, and the $b$-value is 1.20 in the range $12<N<384$. We may expect the range to extend further as in the diamond lattice model.

The difference in $a$-values between the diamond lattice model and the GT-model is attributed to the difference in thickness due to the existence or nonexistence of the hydrogen atoms.

The result on the modified GT-model have a larger fluctuation and we cannot give it a different character from that of GT-model.

\section{Attrition Coefficient}

The nature of the intersections accompanying the dimerization is expressed by Alexandrowicz ${ }^{8}$ to the attrition coefficient

$$
\frac{W_{N}}{\Omega}=c N^{-k}
$$

where $W_{N}$ is the number of samples remaining after dimerization of $N / 2$ step-chains, and $\Omega$ is the number of trial dimerizations. Our results for the attrition coefficient (Table IV) suit this equation. In Figure 3 the results for the attrition

\begin{tabular}{|c|c|c|c|c|c|}
\hline \multicolumn{6}{|c|}{ GT-Model of polyethylene } \\
\hline$N$ & 24 & 48 & 96 & 192 & 384 \\
\hline$W_{N} / W_{N / 2}$ & 0.68 & 0.63 & 0.57 & 0.52 & 0.42 \\
\hline \multicolumn{6}{|c|}{ Modified GT-model of polyethylene } \\
\hline$N$ & 24 & 49 & 96 & 192 & 384 \\
\hline$W_{N} / W_{N / 2}$ & 0.69 & 0.65 & 0.57 & 0.43 & 0.47 \\
\hline \multicolumn{6}{|c|}{ Diamond lattice model } \\
\hline$N$ & 16 & 32 & 64 & 128 & 256 \\
\hline$W_{N} / W_{N / 2}$ & 0.87 & 0.78 & 0.71 & 0.62 & 0.56 \\
\hline$N$ & 512 & 1024 & 2048 & 4096 & 8192 \\
\hline$W_{N} / W_{N / 2}$ & 0.50 & 0.46 & 0.40 & 0.36 & 0.27 \\
\hline
\end{tabular}

Table IV. Values of attirition coefficient coefficients $\log \left(W_{N} / W_{N / 2}\right)$ of the 4-choice cubic lattice by Alexandrowicz and the diamond lattice model and the GT-model of polyethylene are shown to be proportional to $\log N$. These three sets of data produce three parallel lines. The constant $k$ is 0.165 in this calculation. So the difference in model only affects the constant

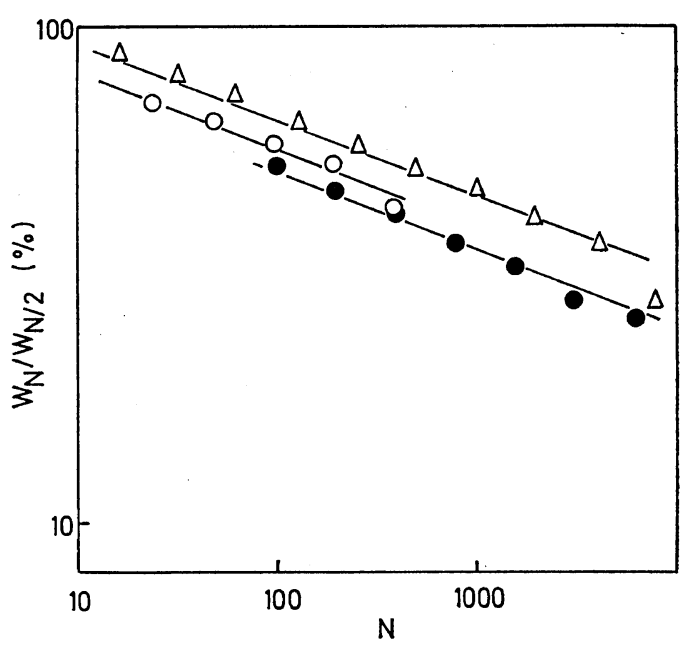

Figure 3. Values of $\log \left(W_{N} / W_{N / 2}\right)$ vs. $\log N:(\triangle)$, diamond lattice model; ( $\bigcirc), \mathrm{GT}$-model; (O), cubic lattice model. 8

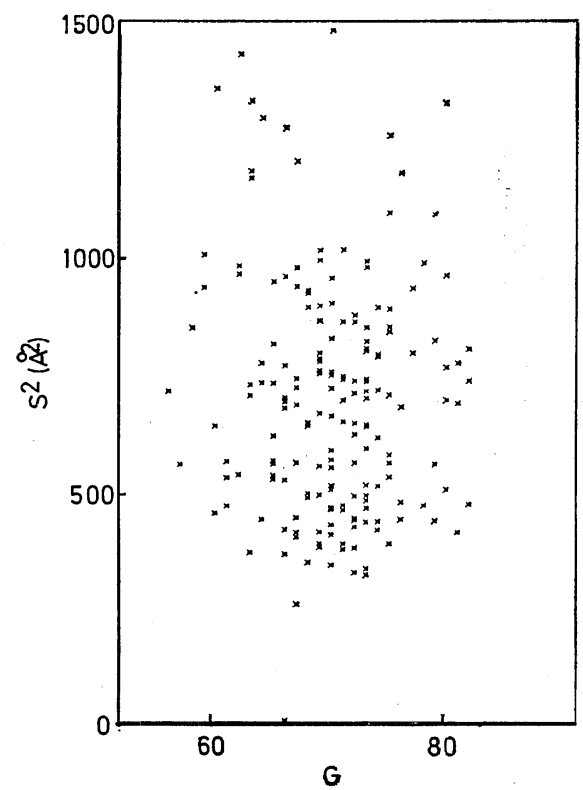

Figure 4. Plots of $S^{2}$ against the number of gauche forms $G$, at $N=192$ of GT-model. 


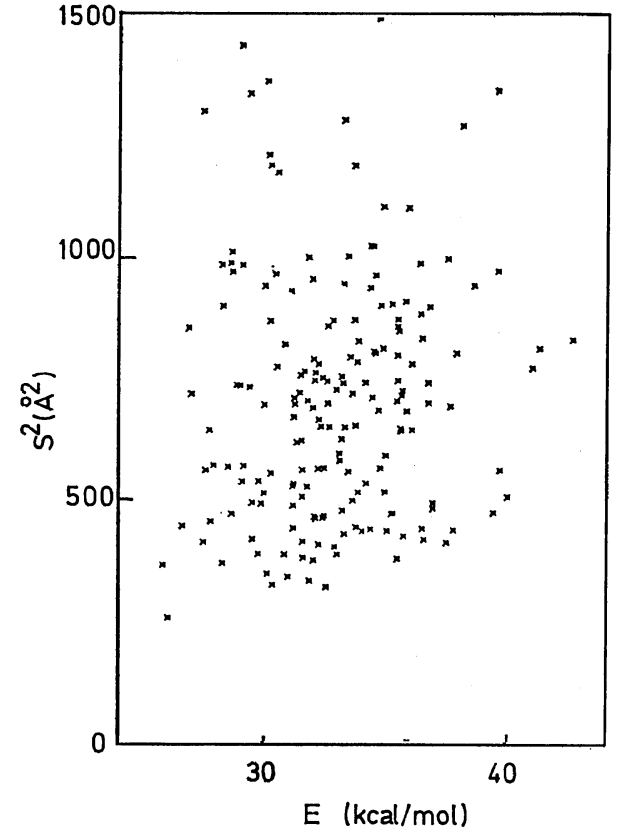

Figure 5. Plots of $S^{2}$ against the intramolecular energy $E$, at $N=192$ of GT-model.

$c$, and the constant $c$ depends primarily on the short-range interaction. The long-range interaction affects largely the constant $k$, and seems to be the same for any model.

\section{$R^{2}, S^{2}, G$ and $E$}

Figure 4 shows that the dimensions of a chain molecule, rich in gauche forms $G$, are not necessarily smaller than those of the trans-rich molecule. The intramolecular energy $E$ has no correlation with $S^{2}$ as shown in Figure 5. The variation in $R^{2}$ with $S^{2}$ is shown in Figure 6. In the case of a random flight chain, the relation between $\left\langle S^{2}\right\rangle_{R}$ and $R^{2}$ is expressed by Hermans and Overbeek. ${ }^{9}$

The mean-square radius of gyration $\left\langle S^{2}\right\rangle_{R}$ with $R$ fixed

$$
\left\langle S^{2}\right\rangle_{R}=\frac{1}{12} N a^{2}\left(1+\frac{R^{2}}{N a^{2}}\right)
$$

and in the case of unperturbed chain

$$
\left\langle S^{2}\right\rangle_{R}=\frac{1}{12}\left\langle R^{2}\right\rangle\left(1+\frac{R^{2}}{\left\langle R^{2}\right\rangle}\right)
$$

For the free rotation of fixed bond angles $\theta$

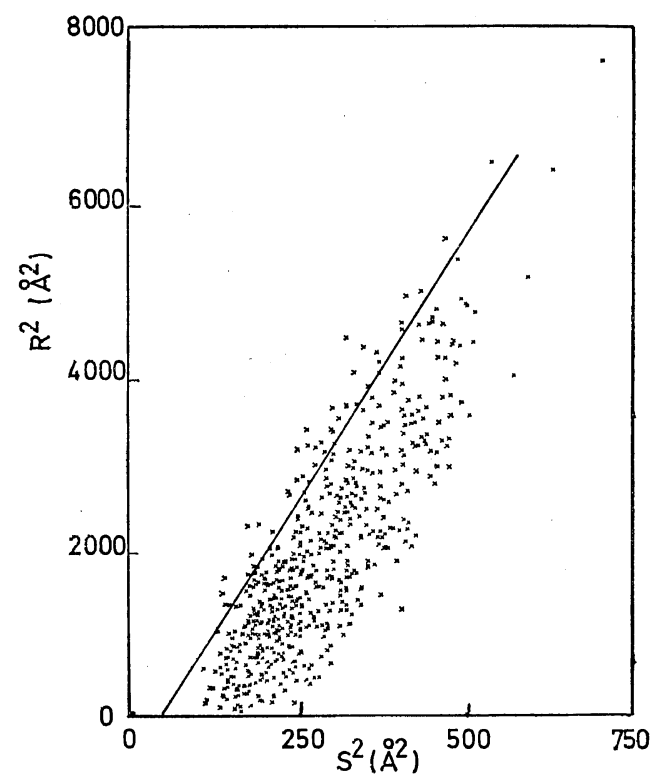

Figure 6. Plots of $R^{2}$ against $S^{2}$, at $N=96$ of GT-model.

$$
\left\langle R^{2}\right\rangle=\frac{1-\cos \theta}{1+\cos \theta} a^{2} N
$$

This relation is shown as the solid line in Figure 6. These figures would give valuable information about the characteristics of chain molecules.

The Monte Carlo calculation for the GT-model of polyethylene in the present scheme cannot be extended further due to the heavy requirement of computer time-for example, 100 walks of $N=192$ required $4 \mathrm{hr}$. As we would have longer chains in the realistic model, which included the intramolecular energy, we should have a new method to make a walk.

\section{REFERENCES}

1. F. T. Wall and J. J. Erpenbeck, J. Chem. Phys., 30, 634 (1959).

2. K. Suzuki, Bull. Chem. Soc. Japan, 41, 538 (1968).

3. S. Windwer, "Markov chains and Monte Carlo calculations in Polymer Science," G. G. Lowry, Ed., Marcel Dekker, N.Y., 1970 p. 126.

4. E. Loftus and P. J. Gans, J. Chem. Phys., 49, 3828 (1968).

5. K. Suzuki and Y. Nakata, Bull. Chem. Soc. Japan, 43, 1006 (1970). 
Effect of Intramolecular Energy on Conformation

6. R. A. Scott and H. A. Scheraga, J. Chem. Phys., $\quad$ 8. Z. Alexandrowicz, J. Chem. Phys., 51, 561 45, 2091 (1966). (1969).

7. S. F. Edwards, Proc. Phys. Soc. (London), 85, 9. J. J. Hermans and J. T. G. Overbeek, Rec. Trav. 613 (1965). Chim., 67, 761 (1943). 